\title{
GESTIÓN INSTITUCIONAL, INVOLUCRAMIENTO DOCENTE Y DE PADRES DE FAMILIA EN ESCUELAS PÚBLICAS DE MÉXICO ${ }^{1}$
}

\author{
Carlos Acevedo ${ }^{2}$ \\ Giovanna Valenti ${ }^{3}$ \\ Eduardo Aguiñaga ${ }^{4}$
}

\begin{abstract}
RESUMEN
La presente investigación se centra en el estudio de los efectos que tienen el involucramiento docente, el involucramiento de padres familia y la gestión institucional en el logro escolar. Para su realización se trabajó con las bases de datos de la Evaluación Nacional de Logros Académicos en Centros Escolares (prueba ENLACE 2012) y el cuestionario CONTEXTO 2012 de alumnos de cuarto y sexto grado de escuelas generales (públicas) de México, controlando por variables socioeconómicas y culturales. El análisis constató que tanto el involucramiento docente como el de padres de familia afectan positivamente el logro académico, siendo el involucramiento docente una de las variables escolares con mayor influencia. Por su parte, ninguna de las variables de gestión institucional resulta significativa. Al indagar si la gestión institucional y el interés de padres de familia afectan los logros escolares por medio de elevar el involucramiento docente, se aprecia que mientras el mayor interés de los padres de familia eleva el involucramiento docente, únicamente los beneficios individuales otorgados a los docentes tienen este efecto. Se concluye con una reflexión en torno a la no significancia de las restantes variables de gestión institucional que, de acuerdo con la literatura especializada, son centrales en la mejora escolar.
\end{abstract}

Palabras clave: estatus socioeconómico y cultural, gestión institucional, involucramiento docente, involucramiento de padres de familia, logro escolar.

1 Este artículo forma parte de la investigación titulada "Análisis de alternativas de gestión escolar para superar los efectos de la desigualdad social en el logro educativo", proyecto 257338 financiado por el Fondo de Investigación Básica SEP-Conacyt.

2 Facultad Latinoamericana de Ciencias Sociales (FLACSO), Ciudad de México, México. Contacto: cacevedo@flacso.edu.mx

3 Departamento de Política y Cultura, Universidad Autónoma Metropolitana UAM-Xochimilco, Ciudad de México, México. Contacto: gvalenti@correo.xoc.uam.mx

4 Unidad de Normatividad y Política Educativa, Instituto Nacional para la Evaluación Educativa, INEE, Ciudad de México, México. Contacto: eaguinaga@inee.edu.mx 


\title{
INSTITUTIONAL MANAGEMENT, TEACHER AND PARENTAL INVOLVEMENT IN PUBLIC SCHOOLS IN MEXICO
}

\begin{abstract}
This research focuses on the effects of teacher and parental involvement and institutional management on student academic performance. This work was based on information from the databases of the National Assessment of Academic Achievement in School Centers (ENLACE 2012 exam) and the questionnaire CONTEXT 2012 applied to fourth and sixth graders in Mexican public schools, controlling for socioeconomic and cultural variables. The analysis finds that both teacher and parent involvement produce a positive influence on student academic performance, with teacher involvement as the most important school influence. On the other hand, none of the institutional management variables is significant. Upon exploring whether institutional management and parental interest affect student achievement through increasing teacher involvement, it was observed that while greater parental interest raises teacher involvement, only those individual benefits granted to the teachers have this effect. This study concludes with a reflection on the non-significance of the remaining institutional management variables that, according to the literature are central to school improvement.
\end{abstract}

Keywords: institutional management, socioeconomic and cultural status, student academic performance, teacher involvement, parental involvement.

\section{Antecedentes}

Desde los años sesenta es posible constatar la fuerte asociación entre estatus socioeconómico (medido a través de diversos recursos económicos, sociales y culturales) y resultados educativos, especialmente a partir del informe conocido como el estudio Coleman (1966). En los años siguientes, el análisis en torno a esta relación se profundizó en investigaciones como las de Bourdieu \& Passeron (1970), Bernstein (1971), Heath (1983), Reynolds \& Walberg (1992), Watkins (1997), Treviño y Treviño (2004).

Dichos estudios confirmaron que los resultados escolares se correlacionan altamente con el estatus socioeconómico de las familias de los estudiantes. A partir de estos hallazgos surgieron cuestionamientos acerca de la influencia de la escuela para conseguir aprendizajes efectivos de los estudiantes provenientes de sectores desaventajados.

En el marco de estas interrogantes, actualmente la Organización para la Cooperación y el Desarrollo Económicos (OCDE) ha destacado que no solo las escuelas que atienden a sectores 
desfavorecidos "tienden a reforzar las desigualdades socioeconómicas de los estudiantes", es decir, "no mitigan el impacto negativo de los estudiantes de entornos desfavorecidos", sino que "amplifican su efecto negativo en su rendimiento" (OECD, 2012, p. 107).

Tal configuración de la desigualdad educativa se agrava en México, en tanto el sistema educativo nacional cuenta con un número importante de lo que dicha organización denomina escuelas en desventaja, a saber, escuelas cuyas composiciones sociales muestran una fuerte presencia de alumnos con bajo nivel socioeconómico y que obtienen resultados educativos más bajos en comparación con los alumnos provenientes de familias con mayores recursos (Salazar, Flores, Florez, Luna y Valenti, 2010; Tapia y Valenti, 2015).

En este contexto, de acuerdo con las bases de datos Excale 2007 y 2010, se aprecia que "las poblaciones socialmente más desfavorecidas obtienen consistentemente menores promedios que las que se encuentran en mejores condiciones" (INEE, 2014, p. 84). La misma tendencia se expresa en los datos de la prueba PISA 2012 (INEE, 2014).

Entre otros elementos, esta situación significa que las desigualdades sociales configuran condiciones de vida y de estudio opuestas entre los diversos grupos de estudiantes. Como ejemplo, se constata que para el caso mexicano: "los estudiantes del cuartil 1 (más bajo) se caracterizan porque sus padres tienen como máximo nivel de estudios la primaria; en casa disponen en promedio de apenas 10 libros, no tienen un cuarto propio ni computadora y no disponen de línea telefónica fija, ni de auto; sin embargo cuentan con una televisión y teléfono celular. Los estudiantes del cuartil 4 (más alto), tienen padres cuyo nivel mínimo de estudios equivale a licenciatura; además en casa cuentan con más de 100 libros, tienen un cuarto propio y más de una computadora con Internet. Sus hogares disponen en promedio de dos televisiones con servicio de cable, tienen línea telefónica fija, al menos tres celulares y más de un auto" (INEE, 2014, p. 95)

"Los cuartiles se construyeron a partir del índice de estatus socioeconómico y cultural de PISA denominado ESCS, por sus siglas en inglés, el cual se integra por el máximo nivel educativo y ocupacional de los padres, y número de posesiones en el hogar" (INEE, 2014, p. 95 , nota 7 ). 
No obstante, la situación descrita no significa que los resultados escolares estén totalmente determinados por los recursos sociales, económicos y culturales que poseen las familias de los estudiantes. En América Latina el efecto promedio de la escuela en los aprendizajes tiende a bordear el 30\% (Blanco, 2009). Para el caso de México, "la contribución de la escuela constituye entre el $27 \%$ y el 34\% del rendimiento mostrado por los alumnos" (Salazar et al., 2010, p. 48).

Por otra parte, estudios centrados en los factores que se desarrollan al interior de las escuelas constatan la importancia de la gestión institucional y de la enseñanza en el aula para lograr buenos resultados educativos a nivel general y para aminorar el impacto negativo de los factores socioeconómicos en el caso de las poblaciones desfavorecidas. En estos estudios se subraya la importancia de la capacidad institucional para definir proyectos educativos consensuados, el uso de información y el trabajo colegiado así como el compromiso docente en el proceso de enseñanza-aprendizaje.

Estas investigaciones están a la base de las reformas educativas llevadas a cabo en el mundo occidental, incluyendo a México. Se conocen como reformas de segunda generación (Fernández, 2004) y se caracterizan por promover cambios hacia dentro de la escuela. En este sentido, y buscando potenciar los efectos que la escuela tiene en los logros escolares, "se promueven políticas que otorgan mayor autonomía y poder a directores; se realizan cambios en el currículo; en la formación inicial y capacitación de profesores" (Martinic, 2010, p. 32).

Para el caso de México, en un contexto de elevada desigualdad socioeconómica y de un sector educativo público que atiende en gran parte a poblaciones desfavorecidas, estas reformas comenzaron a aplicarse a partir de 1992 con la firma del Acuerdo Nacional para la Modernización de la Educación Básica (ANMEB). En ese año se dio comienzo a un proceso de descentralización en el que el gobierno federal impulsó la capacitación y actualización de los docentes con el sistema de Carrera Magisterial, la reforma 
pedagógica curricular y la introducción de elementos relacionados con la gestión y organización de la escuela. En este marco, se desarrollaron programas que tuvieron como eje a los docentes, y a partir del año 2000 se dio un giro en la política educativa que colocó a la escuela y a lo pedagógico en el centro ${ }^{6}$, al mismo tiempo que se puso énfasis en la autonomía y racionalización de la gestión institucional (Acevedo y Valenti, 2015).

En este período fue emblemático el Programa de Escuelas de Calidad (PEC), cuyo objetivo consistió en fortalecer la escasa autonomía que tenían las escuelas públicas para definir e implementar planes de trabajo, ante lo cual se impulsó la elaboración por parte de cada escuela de un Plan Estratégico de Trabajo (PET). Con el PET se les habilitó para diseñar y poner en marcha proyectos educativos propios y también para fortalecer las tareas de acompañamiento y control directivo sobre los docentes. Asimismo, se impulsó la integración de los padres de familia en los proyectos escolares. De este modo se buscó promover el liderazgo institucional del director y su involucramiento en el proceso de enseñanza-aprendizaje (Bracho, 2009; Fierro, Tapia y Rojo, 2009; Rubio, 2011).

En sintonía con este programa se avanzó también en la producción de información escolar con la instauración de un sistema de evaluación de las prácticas y resultados escolares a cargo de la Secretaría de Educación Pública (SEP), de modo que en el año 2002 se creó el Instituto Nacional de Evaluación Educativa (INEE); en el año 2005 se dio origen a la Unidad de Planeación y Evaluación de

6 Una vez que se logró el ANMEB, desde la política educativa se pusieron en marcha diversos programas orientados al mejoramiento del profesorado, el más destacado de estos en el nivel básico fue el Programa Nacional para la Actualización Permanente de los Maestros de Educación Básica en Servicio (PRONAP). Al iniciar el siglo XXI "fueron desarrollados programas de intervención que buscaron incidir en el currículo y en el funcionamiento regular organizativo curricular de las escuelas de educación básica. En el primer caso se ubicaron, por ejemplo, el Programa Nacional de Lectura, las Bibliotecas de Aula, los programas curriculares de Formación Cívica y Ética. En el segundo caso, el Programa de Integración Educativa y la reforma de la organización de las escuelas de educación especial, a partir de la creación de las Unidades de Servicios de Apoyo a la Educación regular (USAER) y el establecimiento de los Centros de Atención Múltiple (CAM)" (Fierro, Tapia y Rojo, 2009, p. 17). 


\section{Políticas Educativas (UPEPE); y en el año 2007 se inició la aplicación de la prueba ENLACE ${ }^{7}$.}

Continuando con este proceso, en 2013 se estableció una reforma educativa que tuvo como eje el reconocimiento del mérito y compromiso profesional de los docentes respecto de promover una educación de calidad y equidad. Así, se subrayó la importancia del rol del docente no solo en términos de su aporte a una educación de calidad, sino que también en disminuir los efectos de los condicionamientos socioeconómicos en los resultados escolares de las poblaciones desfavorecidas. Con este objetivo se substituyó la Carrera Magisterial por el Servicio Profesional Docente (SPD), con lo que se estableció un nuevo modelo de evaluación que asocia los resultados con el ingreso, permanencia, formación e incentivos al profesorado ${ }^{8}$.

7 Examen estandarizado para evaluar el logro educativo que se realiza a los alumnos de cuarto, quinto y sexto de primaria. Además, durante la prueba se aplica un cuestionario que contiene diversas preguntas respecto de variables escolares y familiares. Este cuestionario, se denomina CONTEXTO y se aplica a una muestra representativa de alumnos de cada grado así como a los padres de estos, a cada profesor de los grupos que realiaron la prueba y al director de cada establecimiento en donde esta se hizo. Como se verá más adelante en este estudio se utiliza la base de datos de esta prueba del año 2012. Debido a las diversas controversias que suscitaba este examen, tales como la realización de trampa en el examen por parte del profesor en aras de recibir el incentivo económico que se le otorgaba cuando sus alumnos salían bien evaluados; el INEE y la SEP "acordaron suspender su aplicación en educación básica durante el año 2014 a fin de revisar su diseño y validez" (Tapia y Valenti, 2015, p. 42). En el año 2015 esta prueba fue suplantada por la prueba Plan Nacional para la Evaluación de los Aprendizajes (PLANEA). PLANEA es un examen estandarizado que se realiza en tres modalidades: Evaluaciones de Logro del Sistema Educativo Nacional, Evaluaciones de Logro en las Escuelas y la Evaluación Diagnóstica Censal.

8 Las evaluaciones del Servicio Profesional Docente (SPD) corresponden a un conjunto de evaluaciones para el acceso, medición del desempeño y la promoción de los docentes. En estas se consideran conocimientos del curso a impartir, la pedagogía y la didáctica. La evaluación de desempeño se realiza cada cuatro años. Entre las diferencias que contiene el SPD frente a Carrera Magisterial está la obligatoriedad de los docentes de presentarse a las evaluaciones con el objetivo de instalar la lógica meritocrática en su desempeño. Carrera Magisterial tenía como objetivo central mejorar las condiciones de los docentes en ingresos y formación y no era obligatoria. Si bien en 2011 se firmó un acuerdo entre la Secretaría de Educación Pública SEP y el Sindicato Nacional de Trabajadores de la Educación para que se aplicara la evaluación universal a docentes y directivos del nivel educativo básico con carácter obligatorio, este carácter compulsivo no se traducía en condicionamientos para los docentes que no aprobaran la evaluación, se trataba solo de un ejercicio diagnóstico. En contraste con la reforma educativa de 2013, se definieron condiciones para los docentes o directivos que no aprobaran los exámenes de ingreso o desempeño con la consecuencia de que no fueran incorporados al sistema educativo, o incluso ser separados de la labor docente o directiva si la valoración negativa se repite. 
Como parte de las transformaciones se le otorgó mayor autonomía al INEE en relación con la emisión de lineamientos para la preparación de los exámenes de ingreso y permanencia de los profesores.

En consecuencia, con las reformas de segunda generación se siguió un camino en que "si las escuelas hacen la diferencia y estas dependen en alguna medida de las decisiones organizacionales tomadas en la escuela, el actor docente vuelve a estar en el centro de la enseñanza" (Fernández, 2004, p. 400).

Frente a los retos que impone la promoción de la calidad y equidad por parte del Sistema Educativo Público en México -y en el marco de la implementación de una serie de reformas de segunda generación- es necesario elaborar estudios de carácter nacional en los que, controlando por factores sociales, económicos y culturales (entre otros), se dé cuenta del efecto que tienen en los aprendizajes las variables de gestión institucional y de enseñanza en el aula que han sido promovidas en las últimas reformas. La presente investigación apunta en esta dirección.

\section{Marco conceptual y analítico}

Como ya se adelantó, las reformas de segunda generación se nutrieron de una serie de estudios que subrayan la gestión institucional y la enseñanza del docente en el aula. Estos estudios se desarrollaron en forma paralela.

En lo que respecta a la importancia de la gestión institucional, los análisis se llevaron a cabo en escuelas atípicas que, a pesar de atender a sectores desfavorecidos, logran aminorar de forma importante el impacto de los factores socioeconómicos en el aprendizaje. Estas escuelas se conocen como "escuelas eficaces" o "escuelas efectivas". El estudio de ellas cobró forma en el movimiento de escuelas eficaces (actualmente también conocido como school effects research) y se centró principalmente en las propiedades institucionales de las escuelas que están a cargo del director (y de su equipo directivo, en caso de haberlo). 
Sobre esta base, se han constatado factores o variables de gestión institucional que son responsabilidad del director y que afectan positivamente los resultados escolares, tales como: promoción de trabajo colaborativo, consensuar objetivos comunes de aprendizaje, seguimiento y monitoreo del quehacer docente, procedimientos administrativos basados en la generación y procesamiento sistemático de información, colaboración con padres de familia, entre otros (Bracho, 2009; Brunner \& Elacqua, 2003; Elmore, 2010; Fierro et al. 2009; Fullan 2010; Fuller \& Clarke, 1994; LLECE, 2000; PISA, 2000; Scheerens, 2000; Schmelkes, 1997; Treviño y Treviño, 2004).

Sintetizando la literatura especializada (Coburn \& Rusell, 2008; Elmore, 2010; Fullan, 2010; Hattie, 2009; Lee \& Smith, 1996; Malone, 2011; Unicef y Mineduc, 2004; Treviño y Treviño, 2004), se pueden identificar tres dimensiones de gestión institucional directiva:

- La primera apunta a aquellas acciones de gestión que de forma directa involucran al director en la evaluación y en el monitoreo de las actividades de enseñanza y aprendizaje que lleva a cabo el docente al interior del aula, tales como observación de la clase y evaluación de su planeación y ejecución.

- El segundo conjunto de variables de gestión, corresponde a aquellas que deben tener como eje lograr que los agentes escolares se reúnan entre ellos, discutan y lleguen a acuerdos respecto de temas pedagógicos, especialmente en relación con cómo los estudiantes aprenden, sus dificultades y avances en este proceso. Tales variables serían fundamentales para la construcción de una visión compartida de cómo actuar en aras de la mejora escolar en tanto tengan como foco "un análisis centrado en las relaciones de causa y efecto entre lo que hacemos para influir sobre los procesos de enseñanza-aprendizaje y su efecto real sobre lo que los estudiantes saben hacer" (Elmore, 2010, p. 13). Se trata de actividades que favorecen la construcción de acuerdos que tienen su anclaje en la reflexión conjunta sobre la práctica en el aula, de modo que tales relaciones se hallan sujetas a verificación, rechazo o refinamiento (Elmore, 2010). Si bien en esta dimensión se subraya la importancia de generar una visión escolar compartida entre docentes, también se enfatiza la promoción institucional de la participación de padres de familia en tal visión. En esta línea, en investigaciones llevadas 
a cabo en América Latina (Unicef y Mineduc, 2004), y con base en la propia experiencia en el trabajo de campo con escuelas mexicanas, se ha constatado la especial relevancia que tiene para el logro escolar que la gestión institucional promueva la asistencia de los padres de familia a las juntas escolares.

- Por último, un tercer conjunto de variables de gestión institucional corresponde a aquellas de carácter más organizacional, tales como ascensos docentes y control de asistencia. Se trata de acciones de gestión organizacional o administrativa que se basan en la generación y procesamiento sistemático de información.

Paralelamente al movimiento de escuelas eficaces, se desarrolló otra serie de investigaciones que se concentraron en el análisis de los procesos de enseñanza y aprendizaje en el aula que ocurren en escuelas que logran buenos resultados académicos. Estos estudios no se remiten únicamente a las escuelas efectivas y en "conjunción con la emergente investigación didáctica, dieron lugar a la investigación sobre la efectividad del profesorado o teacher effectiveness research" (Reynolds \& Walberg, 1994; De Corte, 1996; Slavin, 1996 citados en Fernández, 2004, p. 383).

Los estudios de enseñanza efectiva (o de docentes efectivos) se han concentrado en aquellas actividades que desarrolla el docente, que ocurren en el mismo proceso de enseñanza-aprendizaje al interior del aula y que afectan positivamente el logro escolar de los estudiantes (Winters, 2012). Entre estas destacan: planificación de las clases, generar un ambiente de disciplina, conocer la situación particular de cada estudiante respecto de su avance en el proceso de aprendizaje, preocupación para que estos se motiven, aprendan, se expresen y encuentren sentido al conocimiento que se busca entregar, y llevar a cabo un feedback o retroalimentación constante para lograr los objetivos de aprendizaje que se han hecho explícitos a los estudiantes (Sammons, 2001; Scheerens \& Bosker, 1997; Murillo, 2003 citados en Unicef y Mineduc, 2004, p. 25).

El conjunto de factores de enseñanza efectiva corresponden a las variables escolares con mayor impacto en los resultados educativos, especialmente aquellas que dan cuenta de la retroalimentación, 
pues entre mayor el reto o la exigencia, mayor necesidad de retroalimentación por parte del docente: una retroalimentación que tiene como eje "asegurarse de que el aprendiz está en el camino correcto para cumplir con los desafíos" (Hattie, 2009, p. 38). Así, evaluar al estudiante y luego otorgar una retroalimentación oportuna y adecuada ha sido enfatizado como el aspecto más efectivo de enseñanza en diversos estudios (Sheerens \& Bosker, 1997).

Estas actividades de enseñanza-aprendizaje efectivas en el aula darían cuenta de lo que actualmente se puede denominar involucramiento del docente en el proceso de enseñanza-aprendizaje (Hanushek, 1992; Rivkin, 2005; Sanders \& Rivers 1996; Schacter \& Thum, 2003).

Respecto de la relación existente entre factores de gestión institucional y de enseñanza efectiva destacan las investigaciones realizadas por Fullan y Elmore en Estados Unidos, Canadá y América Latina. Con base en estos análisis, es posible afirmar que los elementos de gestión institucional eficaces, lo son en tanto no están presentes de forma separada, sino que actúan en conjunto, convocan a los distintos agentes escolares y tienen como objetivo influir e influyen (precisamente) en lo que se conoce como núcleo pedagógico, a saber: el proceso de enseñanza-aprendizaje que ocurre al interior del aula (Fullan, 2010). En estas investigaciones se da cuenta que la gestión institucional y el liderazgo que esta conlleva, cuando es efectivo, se basa en la utilización estratégica y de forma sistémica de factores-acciones de gestión institucional que impactan en el proceso de enseñanza-aprendizaje que ocurre al interior del aula. En consecuencia, se puede decir que la gestión institucional efectiva sería aquella que impacta en el aula, entre otros elementos (como el currículo), promoviendo el involucramiento docente.

En esta línea es importante subrayar que la gestión institucional efectiva se sustenta en la construcción de un consenso colectivo, de un proyecto educativo compartido por los diversos agentes escolares, en el que se logra legitimar el monitoreo de "la práctica pedagógica de manera más o menos continua", y "la creación de estructuras, procesos y normas organizacionales que vuelven transparente la 
docencia, de manera que esta pueda ser analizada y modificada en función de la retroalimentación respecto de sus efectos" (Elmore, 2010 , p. 76) $)^{9}$. Sin esta construcción colectiva, en que se superan definiciones individualistas acerca de cómo impactar positivamente en el proceso de enseñanza-aprendizaje, la gestión institucional no tendrá el impacto esperado en los resultados escolares (Elmore, 2010; Malone, 2011).

Junto con estas dimensiones y variables relevantes en la consecución del logro escolar, se ha subrayado la necesidad de considerar la influencia que pudieran tener los sistemas de incentivos económicos nacionales de docentes debido a que son importantes como medio de gestión institucional para promover mayor dedicación de los docentes (Hattie, 2009). Al respecto, en México se desarrolló el sistema denominado Carrera Magisterial, que tenía como objetivo central mejorar las condiciones de los docentes en ingresos y formación. Si bien este sistema fue eliminado con la reforma de 2013 y corresponde a una variable de gestión institucional externa a la escuela, es de interés explorar con evidencia si este se justificaba en términos de logro escolar ${ }^{10}$.

Por otra parte, como una variable importante en los resultados escolares, destaca el involucramiento de los padres de familia en el proceso de enseñanza-aprendizaje que llevan a cabo sus hijos o pupilos (Unicef y Mineduc, 2004). Este involucramiento consiste en actividades como estar al pendiente de las calificaciones y asistir a las juntas escolares. Si bien este involucramiento puede estar relacionado con la gestión institucional, en la base de datos no existe información para realizar tal inferencia.

9 Desde la línea de investigación sobre escuelas inclusivas que analiza el peso explicativo que pueden tener las políticas antisegmentación en los aprendizajes, se confirma que la efectividad de estas políticas depende también de la relación virtuosa entre gestión directiva y enseñanza en el aula (Ortiz, 2015). En el estudio del éxito escolar en escuelas norteamericanas, también se confirma este fenómeno (Malone, 2011).

10 En este debate, Fuentes ha argumentado que: "en el año 2013 se han presupuestado 25.000 millones de pesos y el gasto acumulado es de dimensiones monumentales. Sin embargo, el resultado escueto es que no se ha encontrado ninguna correlación positiva entre la calidad de la formación de los alumnos y la pertenencia y jerarquía de sus maestros en el PNCM" (Fuentes, 2013, pp. 31-32). 
Ahora bien, al considerar los efectos cuantitativos de estas dimensiones y variables en el logro escolar (variables de interés) es útil dar cuenta del efecto directo e indirecto que estas tienen en los resultados escolares.

En la literatura especializada es común encontrar investigaciones que indagan únicamente en torno al efecto directo que tienen ciertas variables educativas sobre los resultados escolares. Esto es, se analizan los efectos que estas tienen sobre la variable dependiente (comúnmente logro escolar) sin considerar posibles mediaciones entre estas. Sin embargo, además del análisis del efecto directo de las variables de interés, es necesario recuperar el análisis de los efectos indirectos que presentan principalmente las variables de gestión institucional en el logro escolar. El supuesto que subyace en este enfoque es la dificultad de apreciar efectos directos entre tales variables y logro escolar ${ }^{11}$. Como hemos visto, la gestión institucional para ser efectiva, debe impactar en lo que ocurre al interior del aula o núcleo pedagógico. Sin embargo, a diferencia de lo que podría ocurrir con el involucramiento del docente en el proceso de enseñanzaaprendizaje, tal impacto en el núcleo pedagógico no corresponde a un fenómeno en el que los efectos sean directamente visibles, sino que está inmerso en gran parte en relaciones indirectas y mediatizadas entre las variables. Esta idea se ha corroborado en investigaciones acerca del liderazgo directivo: "la mayoría de los efectos de liderazgo operan indirectamente en la promoción de los resultados escolares por medio de apoyar y mejorar las condiciones de enseñanza y aprendizaje y por su influencia en los profesores y en sus trabajos" (Drago-Severson, 2012; Hallinger \& Heck, 1996; Zepeda, 2012, citados en McCulla y Degenhardt, 2015, p. 559).

Sobre esta base, es que interesa dar cuenta de los efectos indirectos que presentan las variables de gestión institucional en los resultados escolares por medio de elevar el involucramiento docente. Asimismo, interesa mostrar el efecto que tiene en los

11 En términos estadísticos, los efectos indirectos son los que una variable independiente pudiera tener en la variable dependiente a través de otra variable que sirve como mediadora. Es decir, esta variable mediadora podría ayudar a explicar parte de la varianza que nuestra variable independiente de interés tiene sobre la variable dependiente. 
resultados escolares y sobre los profesores el hecho de que los padres se involucren en el proceso de enseñanza-aprendizaje que busca promover la escuela.

Como ya se adelantó, los efectos de las variables de interés deben ser considerados controlando por factores socioeconómicos. Al controlar por estos factores se podrá establecer que dejando constante el efecto promedio de las variables económicas, sociales y culturales de las familias de los estudiantes las variables de interés tienen tal o cuales efectos. Por ende, el efecto de las variables de interés no se confunde con el de otras variables relevantes en los resultados escolares. Asimismo, existe otra serie de variables que tiene lugar al interior de la escuela que debe ser controlada para que su efecto no se confunda con aquellos de las variables de interés, tales como las condiciones de las escuelas, el perfil del estudiante y del docente. En el apartado metodológico se profundiza en las variables de control.

\section{Objetivos e hipótesis}

Utilizando la base de datos ENLACE 2012 y su cuestionario de CONTEXTO, este estudio tiene por objetivo constatar la medida en que los factores de gestión institucional, de enseñanza efectiva y la relación entre estos generan efectos positivos en los resultados de aprendizaje de los estudiantes de educación básica que cursan cuarto y sexto de nivel primaria de México. El mismo análisis se propone respecto del involucramiento de padres de familia y al nivel en el sistema nacional de incentivos Carrera Magisterial. Todo esto controlando por factores socioeconómicos y otras variables escolares que pueden tener un efecto importante en los resultados de los estudiantes.

Para llevar a cabo el análisis planteado, los resultados de aprendizaje de los estudiantes se miden a través del promedio general en español y matemáticas. Asimismo, se trabaja con indicadores de variables de gestión institucional, enseñanza efectiva, involucramiento de padres de familia e incentivos al docente, que están disponibles en ENLACE 2012 y que en la literatura especializada han sido identificados con influencia positiva en el éxito escolar de los estudiantes. 
Se analizan no solo los efectos directos, sino que se profundiza en los efectos indirectos que tienen en el logro escolar las variables de gestión institucional, Carrera Magisterial (variable que se considera parte de la gestión institucional externa a la escuela) e involucramiento de padres de familia. Se supone que este efecto indirecto está mediatizado por el involucramiento docente, por ende, se mide el efecto que estas variables tienen en el involucramiento docente y, por medio de este, en los resultados escolares.

Se manejan las siguientes hipótesis:

- Hipótesis I: entre factores de involucramiento docente y logro escolar existen efectos directos y positivos. Lo mismo ocurre para el caso del involucramiento de los padres de familia.

- Hipótesis II: entre factores de gestión institucional y logro escolar no existen efectos directos.

- Hipótesis III: las variables de gestión institucional afectan positivamente los resultados escolares aunque de forma indirecta, esto es, por medio de elevar el involucramiento docente en el proceso de enseñanza-aprendizaje.

Para analizar los efectos directos e indirectos tal como se ha planteado y para asegurar que estos no se confundan con el efecto de otras variables, no solo se controló por recursos económicos, sociales y culturales de las familias de los estudiantes, sino que como se especifica en la siguiente sección, se controló por otra serie de variables que remiten al perfil del estudiante y del profesor y a las características de las escuelas.

Mediante el tipo de investigación propuesta fue posible realizar un aporte estratégico para otorgar insumos en el diseño de políticas educativas pues en América Latina, especialmente en México, "salvo casos que se destacan por su excepcionalidad, hoy por hoy no se cuenta con estrategias que permitan garantizar resultados de calidad en contextos de exclusión" (López, 2006, p. 57). 


\section{Decisiones metodológicas}

Para contrastar las hipótesis planteadas se utilizó una serie de modelos estadísticos a fin de procesar información de las bases de datos ENLACE y CONTEXTO 2012. Los resultados de la prueba ENLACE se concentraron en los resultados en el examen y tuvieron un carácter censal. La información proporcionada por CONTEXTO se concentraba en variables del contexto familiar y del proceso escolar de los estudiantes y correspondía a un cuestionario aplicado a una muestra de estudiantes, sus padres, así como a los directores y profesores de escuelas en donde se realizó la prueba ENLACE. El cuestionario fue contestado por 32.535 alumnos de cuarto grado y 31.361 de sexto. Para este estudio se generó una base de datos en la que a la información presente en CONTEXTO se agregaron las variables contenidas en ENLACE, por lo cual se trabajó a nivel muestral.

Este análisis se centró en cuarto y sexto de primaria de la modalidad educativa básica general. Se decidió limitar el análisis a ambos niveles debido a que de este modo es posible considerar los resultados obtenidos como referencia para siguientes estudios que se hagan a partir de la prueba PLANEA. Tal como se plantea en el documento rector de PLANEA, analizar cuarto de primaria es relevante en tanto que "este representa un momento de inflexión en el que los alumnos inician la segunda mitad de su educación primaria" (INEE, 2014, p. 14). Mientras que considerar sexto grado "ofrece un buen indicador de la eficacia del proceso educativo en su conjunto, reconociendo los logros de los alumnos a lo largo de varios años de trabajo en los que se van integrando diferentes aprendizajes que conforman una red compleja de conocimientos, habilidades y competencias" (INEE, 2014, p. 15).

En México, la educación básica (preescolar, primaria y secundaria) para el inicio del ciclo escolar 2012-2013 representaba el $85,4 \%$ de las escuelas (INEE, 2014, p. 47). Además, la participación del sector público es mayoritaria en la educación básica (83,7\% en preescolar, 91,3\% en primaria y $87,8 \%$ en secundaria) (INEE, 2014, p. 47). En la educación primaria se imparte de tercero a sexto grado 
y el $86,69 \%$ de estos estudiantes asisten a la modalidad general (ENLACE 2012), modalidad pública (les siguen las modalidades particular con un $9,08 \%$, indígena con un 3,51\% y Conafe con un $0,72 \%)$. En esta modalidad y para los grados de cuarto y sexto, el $60 \%$ de los estudiantes proviene de familias con un ingreso menor a 3.000 pesos (160 dólares) y el $87 \%$ lo hace de familias que perciben un ingreso menor a 8.000 pesos (428 dólares) (ENLACE, 2012). En consecuencia, el estudio se centra en un sector educativo que es público y que atiende en mayor medida a sectores desfavorecidos.

Las técnicas de análisis empleadas consisten en análisis factorial exploratorio y confirmatorio, análisis de regresión lineal multinivel y análisis por medio de ecuaciones estructurales. La técnica de ecuaciones estructurales (modelos SEM) ha cobrado importancia en los últimos años y tiene la ventaja de permitir conocer las relaciones entre las distintas variables latentes en el modelo, considerando la matriz de covarianzas de las variables observadas, lo que posibilita identificar posibles correlaciones entre las variables. Esta técnica permite medir los efectos indirectos ya comentados. Estas técnicas estadísticas se diseñaron y ejecutaron en los software Stata y Mplus.

\section{Construcción de variables}

Respecto de la enseñanza efectiva o involucramiento docente, en el cuestionario CONTEXTO se les realiza una serie de preguntas a los niños que se pueden incluir como indicadores de los componentes de la enseñanza efectiva, especialmente de la retroalimentación que existe en el proceso de enseñanzaaprendizaje ${ }^{12}$. Con un análisis factorial confirmatorio (CFA

12 Queda el vacío, sin embargo, que a los estudiantes no se les pregunta su opinión acerca de la planeación de las clases que realizan sus profesores. En cambio, sí se les pregunta a los directores si evalúan la planeación de la clase de los profesores y si es un tema que se discute en los Consejos Técnicos Escolares. Generalmente, se tiende a subvalorar la percepción de los niños respecto de temas para los que se supone se requiere tener mayor edad para opinar. Sin embargo, es común que se demuestre que las valoraciones o percepciones que hacen los niños en torno al trabajo de sus profesores, incluyendo la planeación de las clases, sean un excelente predictor de los resultados que obtiene el profesor en diversos exámenes y en lo efectivo que este puede llegar a ser (Hattie, 2009). 


\section{por sus siglas en inglés), tales variables dan forma al índice denominado involucramiento docente ${ }^{13}$ (Tabla 1).}

\section{Tabla 1}

Análisis factorial confirmatorio de involucramiento docente

\begin{tabular}{|c|c|c|c|c|c|}
\hline \multirow[b]{2}{*}{ Variable } & \multirow[b]{2}{*}{ Nombre } & \multicolumn{2}{|c|}{ Cuarto grado } & \multicolumn{2}{|c|}{ Sexto grado } \\
\hline & & Carga & $\begin{array}{l}\text { Desviación } \\
\text { estándar }\end{array}$ & Carga & $\begin{array}{l}\text { Desviación } \\
\text { estándar }\end{array}$ \\
\hline $\begin{array}{l}\text { Ayuda cuando los alumnos no } \\
\text { entienden }\end{array}$ & mayuda & $0,696^{* * *}$ & 0,003 & $0,722^{* * *}$ & 0,003 \\
\hline $\begin{array}{l}\text { Permite que el alumno se } \\
\text { exprese }\end{array}$ & mopinion & $0,713^{* * *}$ & 0,003 & $0,734^{* * *}$ & 0,003 \\
\hline Respeta a los alumnos & mrespeta & $0,731^{* * *}$ & 0,003 & $0,756^{* * *}$ & 0,003 \\
\hline $\begin{array}{l}\text { Toma la opinión de los alumnos } \\
\text { en cuenta }\end{array}$ & mtoma & $0,686 * * *$ & 0,003 & $0,727^{* * *}$ & 0,003 \\
\hline Motiva a los alumnos & mmotiva & $0,643^{* * *}$ & 0,004 & $0,653^{* * *}$ & 0,004 \\
\hline Revisa las tareas & mrevisat & $0,563^{* * *}$ & 0,004 & $0,584^{* * *}$ & 0,004 \\
\hline Mantiene la disciplina & mdisc & $0,63^{* * *}$ & 0,004 & $0,615^{* * *}$ & 0,004 \\
\hline Corrige las tareas & mcorrije & $0,68^{* * *}$ & 0,003 & $0,686^{* * *}$ & 0,003 \\
\hline Deja tareas & mdejatar & $0,599 * * *$ & 0,004 & $0,617^{* * *}$ & 0,004 \\
\hline $\begin{array}{l}\text { Escucha y da recomendaciones } \\
\text { al estudiante }\end{array}$ & mescyrec & $0,575^{* * *}$ & 0,004 & $0,528^{* * *}$ & 0,004 \\
\hline
\end{tabular}

Fuente: Elaboración propia a partir de prueba ENLACE y cuestionario CONTEXTO 201214.

13 El CFA otorga una variable latente, índice o factor que en este caso se denomina involucramiento docente. En la Tabla 1 se aprecian los pesos de cada una de las variables tiene en la variable latente y se prueba si su asociación es significativa. Es pertinente señalar que los CFA se realizaron a través del estimador de Mínimos Cuadrados Ponderados, el cual permite omitir el supuesto de normalidad en la distribución y por tanto resulta apropiado para el cálculo con variables ordinales, variables dicotómicas y variables continuas que no se ajusten a criterios de normalidad. El cálculo resulta de minimizar la función de la diferencia entre la matriz de covarianza empírica y la matriz de covarianza del modelo (Méndez, 2003; Raykov \& Marcoulides, 2006).

$14 * p<0,10 ; * *<<0,05 ; * * *<<0,01$. El " $p$ " ( $\mathrm{p}$-valor) implica la probabilidad de que el valor del coeficiente no corresponda al valor poblacional. De modo que entre menor este valor menor tal probabilidad. También se puede entender en términos de "confianza". Así, entre menor el valor $p$, mayor confianza de que en los intervalos del valor del coeficiente muestral se encuentra el valor poblacional: $p<0,10=90 \%, p<0,05=95 \%, p<0,01=99 \%$. La confianza indica que de 100 muestras que se tomen, $90 \%, 95 \%$ o 99\% de estas, contendrán el valor poblacional. En el mismo sentido se habla que un coeficiente es significativo al 90\%, 95\% o 99\%. Esto aplica para todos los modelos estadísticos presentados en esta investigación. No obstante, es pertinente aclarar que en el caso específico del CFA, el p-valor da cuenta de la probabilidad de que las varianzas de las variables que forman la variable latente no estén correlacionadas en términos del valor poblacional. 
En relación con las variables de gestión institucional, como se presentó en el marco conceptual y siguiendo las investigaciones empíricas realizadas en América Latina, Estados Unidos y Canadá (Coburn \& Rusell, 2008; Elmore, 2010; Fullan, 2010; Hattie, 2009; Lee \& Smith, 1996; Malone, 2011; Mineduc, 2004; Treviño y Treviño, 2004) se identifican tres dimensiones, de modo que: en primer lugar se construyó un índice denominado Evaluación pedagógica, el cual da cuenta de actividades que involucran al director de forma directa en la evaluación y monitoreo de las actividades de enseñanza y aprendizaje. Las variables contempladas así como el peso particular que cada variable tiene en el índice se muestran en la Tabla 2.

Tabla 2

Análisis factorial confirmatorio de evaluación pedagógica

\begin{tabular}{lccccc}
\hline & & \multicolumn{2}{c}{ Cuarto grado } & \multicolumn{2}{c}{ Sexto grado } \\
\hline Etiqueta actividades a evaluar & Variable & Carga & $\begin{array}{c}\text { Desviación } \\
\text { estándar }\end{array}$ & Carga & $\begin{array}{c}\text { Desviación } \\
\text { estándar }\end{array}$ \\
\hline Dominio de los temas & dominio & $0,857^{* * *}$ & 0,002 & $0,863^{* * *}$ & 0,002 \\
Estrategias de enseñanza & estrat & $0,9^{* * *}$ & 0,002 & $0,896^{* * *}$ & 0,002 \\
Planeación de la clase & planea & $0,607^{* * *}$ & 0,004 & $0,637^{* * *}$ & 0,004 \\
Apoyo académico & apoyo & $0,746^{* * *}$ & 0,003 & $0,768^{* * *}$ & 0,003 \\
Métodos de evaluación a los alumnos & métodos & $0,786^{* * *}$ & 0,003 & $0,797^{* * *}$ & 0,002 \\
\hline
\end{tabular}

Fuente: Elaboración propia a partir de prueba ENLACE y cuestionario CONTEXTO 2012.

Un segundo conjunto de variables de gestión institucional corresponde a aquellas que pueden promover el trabajo colegiado y visiones compartidas del proceso de enseñanza-aprendizaje. En esta serie se considera el resultado de los estudiantes del profesor al momento de evaluar su desempeño, la frecuencia de visitas del director a las aulas, la frecuencia de visitas del supervisor a la escuela, la frecuencia de juntas escolares que se realizan con padres de familia, la frecuencia de juntas del Consejo Técnico Escolar y los temas pedagógicos tratados en ellas.

Sobre los distintos temas que se discuten en las juntas de Consejo Técnico Escolar, el análisis se limita a aquellos relacionados con la escuela y con el proceso de enseñanza aprendizaje dentro del 
aula, dejando fuera temas externos a lo pedagógico. Con un CFA se construyó el siguiente índice de temas pedagógicos en las juntas de Consejo Técnico Escolar (Tabla 3).

Tabla 3

Análisis factorial confirmatorio de temas pedagógicos tratados en Consejo Técnico Escolar

\begin{tabular}{|c|c|c|c|c|c|}
\hline \multirow[b]{2}{*}{ Etiqueta } & \multirow[b]{2}{*}{ Variable } & \multicolumn{2}{|c|}{ Cuarto grado } & \multicolumn{2}{|c|}{ Sexto grado } \\
\hline & & Carga & $\begin{array}{l}\text { Desviación } \\
\text { estándar }\end{array}$ & Carga & $\begin{array}{c}\text { Desviación } \\
\text { estándar }\end{array}$ \\
\hline Planeación de actividades & tplanea & $0,491^{* * *}$ & 0,004 & $0,503^{* * *}$ & 0,004 \\
\hline $\begin{array}{l}\text { Estrategias de evaluación de } \\
\text { cumplimiento de planeación }\end{array}$ & testrat & $0,707^{* * *}$ & 0,003 & $0,713^{* * *}$ & 0,003 \\
\hline Planeación de clases & tplaneacl & $0,618^{* * *}$ & 0,004 & $0,643^{* * *}$ & 0,004 \\
\hline $\begin{array}{l}\text { Estrategias de enseñanza } \\
\text { aprendizaje }\end{array}$ & testrate & $0,767^{* * *}$ & 0,003 & $0,773^{* * *}$ & 0,003 \\
\hline $\begin{array}{l}\text { Discusión y análisis de secuencias } \\
\text { temáticas }\end{array}$ & tdisc & $0,75^{* * *}$ & 0,003 & $0,756^{* * *}$ & 0,003 \\
\hline Selección de material didáctico & tselecc & $0,773^{* * *}$ & 0,002 & $0,774^{* * *}$ & 0,003 \\
\hline Intercambio de prácticas escolares & tintercamb & $0,628 * * *$ & 0,004 & $0,62^{* * *}$ & 0,004 \\
\hline $\begin{array}{l}\text { Estrategias de evaluación de } \\
\text { aprendizaje }\end{array}$ & testeval & $0,803^{* * *}$ & 0,002 & $0,809 * * *$ & 0,002 \\
\hline Atención a problemas de aprendizaje & testaten & $0,806^{* * *}$ & 0,002 & $0,809 * * *$ & 0,002 \\
\hline $\begin{array}{l}\text { Estímulos y reconocimiento a } \\
\text { alumnos }\end{array}$ & testest & $0,731^{* * *}$ & 0,003 & $0,733^{* * *}$ & 0,003 \\
\hline $\begin{array}{l}\text { Problemas de disciplina de los } \\
\text { alumnos }\end{array}$ & tprobl & $0,575^{* * *}$ & 0,004 & $0,602^{* * *}$ & 0,004 \\
\hline $\begin{array}{l}\text { Estrategias de actualización y } \\
\text { capacitación laboral }\end{array}$ & testact & $0,679 * * *$ & 0,003 & $0,696 * * *$ & 0,003 \\
\hline
\end{tabular}

Fuente: Elaboración propia a partir de prueba ENLACE y cuestionario CONTEXTO 2012.

Debido a que cada variable seleccionada puede diferir de forma importante en el efecto que tiene en el logro escolar, no se confeccionó un índice del conjunto de estas.

En tercer lugar, como un conjunto de variables que da cuenta de la esfera organizativa, se consideraron los beneficios que reciben los docentes a partir de la evaluación que realiza el director de su desempeño, la evaluación de la asistencia del profesor y de su puntualidad. 
Con la intención de reducir el número de variables que miden una misma dimensión, se recurrió a la construcción de un índice en relación con los beneficios que reciben los docentes (a partir de la evaluación que realiza el director de su desempeño). En la Tabla 4 se muestran las variables incluidas en el índice así como el peso respectivo de cada una de estas.

Tabla 4

Análisis factorial confirmatorio de beneficios económicos o materiales

\begin{tabular}{llcccc}
\hline & & \multicolumn{2}{c}{ Cuarto grado } & \multicolumn{2}{c}{ Sexto grado } \\
\hline Etiqueta & Variable & Carga & $\begin{array}{c}\text { Desviación } \\
\text { estándar }\end{array}$ & Carga & $\begin{array}{c}\text { Desviación } \\
\text { estándar }\end{array}$ \\
\hline Selección de grupo & selgpo & $0,395^{* * *}$ & 0,011 & $0,419^{* * *}$ & 0,011 \\
Permisos especiales & especial & $0,501^{* * *}$ & 0,014 & $0,517^{* * *}$ & 0,013 \\
Reconocimiento en la escuela & recoesc & $0,104^{* * *}$ & 0,009 & $0,092^{* * *}$ & 0,009 \\
Más horas de clase & bmasclases & $0,337^{* * *}$ & 0,011 & $0,349^{* * *}$ & 0,012 \\
Beneficios económicos & becono & $0,097^{* * *}$ & 0,011 & $0,066^{* * *}$ & 0,012 \\
Beneficios en especie & bespec & $0,3^{* * *}$ & 0,012 & $0,304^{* * *}$ & 0,012 \\
Ascenso & escala & $0,183^{* * *}$ & 0,009 & $0,167^{* * *}$ & 0,009 \\
\hline
\end{tabular}

Fuente: Elaboración propia a partir de prueba ENLACE y cuestionario CONTEXTO 2012.

Al igual que el conjunto de variables anterior, las de esta dimensión pueden diferir de forma importante en el efecto que tienen en el logro escolar, por lo cual se propuso constatar el impacto de cada una por separado.

Por otra parte, se agregó al análisis la frecuencia de asistencia de los padres a las juntas escolares (apoyo5) y el interés de estos respecto de las calificaciones de sus hijos o pupilos (apoyo4). Si bien estas variables pueden ser promovidas por el director (y su equipo directivo) (Unicef y Mineduc, 2004) y así ser parte de los componentes de la gestión institucional, en la base de datos que se trabajó no se cuenta con la información para realizar tal inferencia.

Asimismo, como indicadores de incentivos docentes que se dirigen a promover mayor dedicación de estos, se agregó el nivel de Carrera Magisterial en el que el docente se encuentra (cmag). 


\section{Variables de control}

Respecto de las variables a nivel del estudiante se consideraron el sexo (sexol), si sufre de bullying (bullyingl), problemas cognitivos (cognitivo), problemas físicos (físico), maltrato familiar (maltrato), su frecuencia de lectura (freclec), si ha reprobado (reprobo), si cuenta con un hogar con padre o madre o solo con uno de estos (hogar), número de días que trabaja (dtrabajo), primera lengua que aprendió a hablar (lengua2 indígena, lengua3 extranjera, lengua4 español e indígena), expectativas en términos de dónde quiere llegar en su carrera académica o hasta qué nivel desea seguir estudiando (expest).

Respecto de las variables del profesor, se consideraron los años de experiencia que lleva en la escuela (expesc) y número de cursos de actualización que ha tomado (cursoact).

En relación con las variables de condiciones escolares, se consideraron la disposición de equipo y de espacios para la realización de actividades de enseñanza (equipamientol y espaciosl), el número de libros y nivel de exigencia de la escuela (librosesc y exige), y la frecuencia con que se evalúa el logro del estudiante (evallogro). Asimismo, se agregó la variable de temas no pedagógicos tratados en las juntas de Consejo Técnico Escolar (tema_ext).

Respecto de las variables sociales, económicas y culturales que caracterizan a la familia del estudiante, se construyó un índice denominado Estatus socioeconómico y cultural o ESCYC (Tabla 5). 
74 GESTIÓN INSTITUCIONAL, INVOLUCRAMIENTO DOCENTE Y DE PADRES DE FAMILIA EN ESCUELAS PÚBLICAS DE MÉXICO - C. Acevedo, G. Valenti y E. Aguiñaga

Tabla 5

Análisis factorial confirmatorio de estatus socioeconómico y cultural

\begin{tabular}{lllccc}
\hline & & \multicolumn{2}{c}{ Cuarto grado } & \multicolumn{2}{c}{ Sexto grado } \\
\hline Etiqueta & Variable & Carga & $\begin{array}{c}\text { Desviación } \\
\text { estándar }\end{array}$ & Carga & $\begin{array}{c}\text { Desviación } \\
\text { estándar }\end{array}$ \\
\hline Escolaridad de la madre & estmadre & $0,763^{* * *}$ & 0,003 & $0,77^{* * *}$ & 0,003 \\
Escolaridad del padre & estpadre & $0,758^{* * *}$ & 0,003 & $0,76^{* * *}$ & 0,003 \\
Ocupación de la madre & ocupmadre & $0,596^{* * *}$ & 0,005 & $0,603^{* * *}$ & 0,005 \\
Ocupación del padre & ocupadre & $0,761^{* * *}$ & 0,003 & $0,75^{* * *}$ & 0,003 \\
Ingreso & ingreso & $0,764^{* * *}$ & 0,003 & $0,768^{* * *}$ & 0,004 \\
Libros en casa & libros & $0,565^{* * *}$ & 0,005 & $0,549^{* * *}$ & 0,005 \\
\hline
\end{tabular}

Fuente: Elaboración propia a partir de prueba ENLACE y cuestionario CONTEXTO 2012.

Para controlar los problemas de confiabilidad de ENLACE, se agregó una variable que indica el número de exámenes sospechosos que se presentaron en cada grado escolar (nocon4 - nocon6). Por exámenes sospechosos se entiende aquellos cuyas respuestas son iguales en el mismo grado de la misma escuela, por lo que se podría presumir que esta escuela hizo trampa. En aras de robustecer este control, se agregó un término de interacción entre el estatus socioeconómico y cultural con la proporción de exámenes sospechosos (esecxtramp) para impedir que el efecto del estatus socioeconómico y cultural se vea subestimado debido a la trampa realizada en alguna escuela ${ }^{15}$.

Finalmente, para identificar los efectos que el involucramiento docente y el estatus socioeconómico y cultural de la familia del alumno tienen en los resultados de los estudiantes sin que se confundan con el contexto escolar (composiciones socioeconómicas y culturales y el nivel de dedicación docente en las escuelas), se agregaron como variables de control los efectos medios de estas variables (inv_med y esec_med).

15 El efecto del estatus socioeconómico y cultural puede verse subestimado en los casos de las escuelas que presentan exámenes que hacen suponer que hicieron trampa, al permitir a todos los estudiantes obtener un puntaje alto y similar sin importar las características socioeconómicas y culturales de su hogar. Es decir, sus respuestas estarán condicionadas por la trampa y no por sus conocimientos o aprendizajes que se ven afectados por su estatus socioeconómico y cultural. 


\section{Resultados}

De acuerdo con la Tabla 6, en cuanto a los efectos directos cabe destacar que al analizar con un modelo multinivel todas las variables consideradas en el estudio (variables de interés y de control) para cuarto y sexto grado: el estatus socioeconómico y cultural (en adelante ESCYC) es una de las variables explicativas que más peso tiene sobre el resultado de la prueba ENLACE 2012 (17,22 en cuarto y 18,47 en sexto grado). Es decir, a medida que sube en una unidad el nivel del ESCYC de las familias, el resultado de la prueba ENLACE aumenta en aproximadamente 18 puntos para los estudiantes de ambos grados. Este efecto solo es superado cuando el estudiante tiene un problema cognitivo; si la primera lengua que aprendió a hablar es indígena o español; y si ha reprobado. Las últimas dos variables se encuentran altamente correlacionadas con el ESCYC ${ }^{16}$. Así, en sintonía con la literatura especializada, se aprecia que en el sistema de educación primaria general en México, el ESCYC condiciona fuertemente los resultados escolares.

Asimismo, en concordancia con la literatura, se aprecia que de manera general el impacto de la escuela en el logro del estudiante bordea el 30\% (36\% para cuarto y 32\% para sexto grado) ${ }^{17}$. Ahora bien, si se constata la correlación entre el efecto del nivel escuelas (constante del modelo) y el ESCYC, se observa que el efecto de la escuela se ve disminuido al incrementarse el ESCYC de la familia del estudiante. En consecuencia, para los estudiantes de sectores sociales más aventajados el que vayan a tal o cual escuela influye en sus resultados académicos en menor medida que sus homólogos

16 Como es de esperar, el impacto del ESCYC de la familia del estudiante se ve aminorado en las escuelas que hicieron trampa. Esto se puede observar a través del coeficiente del término de interacción de estatus por trampa (esecxtramp). Esta variable es el resultado de multiplicar ESCYC y la proporción de exámenes sospechosos. De esta forma, al incrementarse la proporción de exámenes sospechosos el valor de esta variable se incrementará. Pero si no hay exámenes sospechosos en ese grado el valor será 0 y, por tanto, esta variable es anulada. Por lo que si se quiere saber el efecto del ESCYC en el rendimiento en la prueba se aprecia que este tenderá a disminuir, dado que el coeficiente es negativo, entre más exámenes sospechosos haya. Pero cuando no haya exámenes sospechosos no habrá ningún efecto sobre el ESCYC.

17 En un modelo de regresión multinivel, estos datos vienen dados por el coeficiente de correlación intraclase (ICC por sus siglas en inglés). 
desfavorecidos ${ }^{18}$. Esto se podría entender en tanto gran parte del éxito escolar de los sectores aventajados viene dado por el capital social, económico y cultural que les transmite sus familias y que los aventaja para lograr éxito escolar en contraste con estudiantes que provienen de familias desaventajadas (Bourdieu, 1998), donde por el contrario sí puede marcar una diferencia considerable la escuela a la que se asiste (Nye, Konstantopoulos \& Hedges, 2004) ${ }^{19}$. En consecuencia, existen elementos para señalar que los factores escolares son vitales sobre todo para impactar en los resultados de estudiantes desfavorecidos ${ }^{20}$.

En este sentido y respecto de las variables de interés de esta investigación, para ambos grados y en sintonía con la literatura

18 En sintonía con este hallazgo, en un estudio realizado para las escuelas primarias y medias de Texas, USA, se constata que el impacto de la gestión institucional directiva en los resultados escolares, es mayor en las escuelas "altamente pobres" (Branch, Hanushek \& Rivkin, 2013, p. 65).

19 En relación a las restantes variables de control, de las características de las escuelas se aprecia que el número de libros favorece un mejor aprovechamiento en sexto grado, mientras que en cuarto no tiene un efecto estadísticamente significativo.

De parte del docente, la antigüedad de este favorece (en menor medida) los resultados de sus estudiantes de sexto grado en la prueba. Mientras que el número de cursos de actualización tiene un efecto positivo en los estudiantes de cuarto año.

Dentro de las variables individuales, se observa que las expectativas académicas de los estudiantes, así como la frecuencia con la que leen, son las variables que afectan con mayor fuerza su rendimiento en el examen, siendo en sexto el nivel donde estas presentan una mayor influencia. En relación a si el estudiante sufre de bullying, esta variable afecta en menor medida aunque sigue siendo significativa en el desempeño escolar, de modo que en cuarto grado su efecto es negativo y, extrañamente, para sexto grado se torna positivo. Esto último podría estar indicando que en sexto grado los estudiantes que sufren de bullying son aquellos que se caracterizan por obtener buenos resultados. Relación que requiere de mayores investigaciones para ser corroborada.

Por su parte, que el estudiante presenta problemas cognitivos o haya reprobado anteriormente tiende a afectar negativamente su desempeño en la prueba, sobre todo en el caso de sexto grado. Por otro lado, tener una lengua diferente al español como lengua nativa tiende a afectar de forma negativa el desempeño.

Respecto de la situación familiar, el tener un hogar completo tiene un efecto positivo en el aprovechamiento de los estudiantes. El que haya maltrato de parte de algún miembro de la familia afecta de forma negativa el desempeño del niño.

20 Si se profundiza aún más en esta relación, se tiene que en sistemas educativos altamente segregados (como lo son los sistemas chileno y mexicano), los estudiantes acomodados que asisten a escuelas con mayor componente de estudiantes desfavorecidos, tendrán un resultado escolar menor al que podrían lograr al asistir en escuelas con sus pares. Al contrario, los estudiantes desfavorecidos que asisten a escuelas cuya mayoría de estudiantes pertenecen a estratos favorecidos, elevarán sus resultados escolares en comparación con los que obtendrían en escuelas desfavorecidas (Ortiz, 2015). No obstante, precisamente en los sistemas altamente segregados, no son comunes los tránsitos sociales-educativos señalados, y más bien, la normalidad del funcionamiento del sistema tiende a la reproducción de la segregación. La segregación al interior de las escuelas, es una variable que profundizaremos en próximas investigaciones. 
revisada, la variable que más impacta en el logro escolar corresponde al involucramiento del docente reportado por los estudiantes. El efecto del involucramiento docente (16,04 y 15,03 para cuarto y sexto grado, respectivamente) es muy cercano al del ESCYC, lo cual indica la importancia de la labor y dedicación del docente para aminorar los condicionamientos socioeconómicos. De este modo, se confirma la Hipótesis I en la que se planteaba que existía un efecto directo y positivo entre involucramiento docente y resultados escolares. Con los datos analizados ahora es posible afirmar que el efecto no solo es directo y positivo, sino que también es elevado. Asimismo, aunque con un impacto menor al del docente, se confirma que la participación de los padres en las juntas escolares y la atención que estos ponen en las calificaciones de sus hijos (variables que pueden estar relacionadas con la gestión institucional), poseen un efecto directo positivo y significativo ${ }^{21}$ en los resultados escolares $(6,13$ y 3,23 para cuarto grado y 6,89 y 2,63 para sexto). Si bien llama la atención que la frecuencia de juntas con padres que se realizan en la escuela (parte de la segunda dimensión de gestión institucional) no tiene un efecto estadísticamente significativo, el efecto que tiene el involucramiento de padres de familia da pie para afirmar que promoverlo en el proceso de enseñanza-aprendizaje que se propone la escuela tendrá efectos directos y positivos en los resultados escolares.

En relación con la Hipótesis II, efectivamente las variables de gestión institucional no tienen un efecto directo en los resultados de los estudiantes. No se constata una relación directa y significativa entre estas variables y resultados escolares. Lo mismo ocurre para el caso del nivel en Carrera Magisterial.

21 El tamaño o la dirección positiva o negativa del efecto no implica que este sea significativo o no. Así, aunque sea muy pequeño o negativo, un efecto puede ser significativo, es decir, es muy probable $(90 \%, 95 \%, 99 \%)$ que tenga una influencia sobre la variable dependiente, aunque esta influencia sea muy pequeña y esté relacionada de forma inversa con la variable dependiente. 
78 GESTIÓN INSTITUCIONAL, INVOLUCRAMIENTO DOCENTE Y DE PADRES DE FAMILIA EN ESCUELAS PÚBLICAS DE MÉXICO - C. Acevedo, G. Valenti y E. Aguiñaga

Tabla 6

Resultados de regresión multinivel. Cuarto y sexto grado

\begin{tabular}{|c|c|c|c|c|}
\hline \multirow[b]{2}{*}{ Variable } & \multicolumn{2}{|c|}{ Cuarto grado } & \multicolumn{2}{|c|}{ Sexto grado } \\
\hline & Coef. & Std. Err. & Coef. & Std. Err. \\
\hline Estatus socioeconómico y cultural & $17,22482^{* * *}$ & 0,8824289 & $18,4672^{* * *}$ & 0,9019265 \\
\hline Trampa & $179,6165^{* * *}$ & 15,93425 & $168,6936^{* * *}$ & 18,69678 \\
\hline Término de interacción de estatus por trampa & $-32,79644^{* * *}$ & 6,285914 & $-31,28525^{* * *}$ & 8,752029 \\
\hline Bullying & $-3,609815^{* * *}$ & 0,4173836 & $1,978289 * * *$ & 0,44886 \\
\hline Problemas cognitivos & $-19,18874^{* * *}$ & 1,161781 & $-29,32028^{* * *}$ & 1,331866 \\
\hline Problemas físicos & $-6,305928 * * *$ & 1,234796 & $-3,717693^{* * *}$ & 1,446742 \\
\hline Frecuencia con la que el niño lee & $13,11453^{* * *}$ & 0,6071071 & $14,54533^{* * *}$ & 0,6452891 \\
\hline Expectativas académicas & $11,6786^{* * *}$ & 0,4393378 & $13,30419 * *$ & 0,5256807 \\
\hline Hogar completo & $13,26095^{* * *}$ & 1,223317 & $4,527119 * * *$ & 1,388337 \\
\hline Niño trabaja (días) & $-2,452199 * * *$ & 0,2486023 & $-2,975761^{* * *}$ & 0,3080292 \\
\hline Involucramiento docente & $16,04641^{* * *}$ & 0,6986421 & $15,03188^{* * *}$ & 0,8537694 \\
\hline Primera lengua: lengua indígena & $-27,82035^{* * *}$ & 4,135151 & $-21,0677^{* * *}$ & 6,629382 \\
\hline Primera lengua: lengua extranjera & $-10,91363^{* * *}$ & 4,004507 & $-11,87175^{* *}$ & 5,613153 \\
\hline Primera lengua: español-indígena & $-16,84293^{* * *}$ & 3,849005 & $-13,55677^{* *}$ & 5,446368 \\
\hline Maltrato familiar & $-8,63748 * *$ & 0,3684188 & $-5,041818^{* * *}$ & 0,4992783 \\
\hline Reprobó & $-25,98776^{* * *}$ & 1,684496 & $-37,86446 * * *$ & 1,775559 \\
\hline Sexo & 1,298705 & 1,076307 & $-5,578143^{* * *}$ & 1,16137 \\
\hline Está al pendiente de las calificaciones (padres) & $6,128454^{* * *}$ & 0,5345124 & $6,882851^{* * *}$ & 0,6858497 \\
\hline Asiste a las juntas (padres) & $3,238048 * * *$ & 0,5147382 & $2,625385^{* * *}$ & 0,6494095 \\
\hline Carrera magisterial & $-0,7042868$ & 0,9901795 & 1,872039 & 1,02932 \\
\hline Reuniones de consejo académico & 2,748146 & 2,016182 & 0,2330012 & 1,843993 \\
\hline Equipamiento & $-1,251231$ & 2,26056 & $-0,4102594$ & 2,431078 \\
\hline Suficiencia de espacios & 2,319399 & 1,987516 & 0,6686352 & 2,039306 \\
\hline Frecuencia con que se evalúa el logro & $-0,6139762$ & 2,128338 & 0,940874 & 2,029616 \\
\hline Exigencia de la escuela & $-0,147118$ & 0,5712297 & 0,8754739 & 0,6078413 \\
\hline Frecuencia de juntas con padres & 5,235544 & 3,568951 & 5,200217 & 3,839367 \\
\hline Libros en la escuela & 0,378681 & 1,51456 & $4,47812^{* * *}$ & 1,538653 \\
\hline Temas no pedagógicos en las reuniones de consejo & 0,3676875 & 2,371175 & $-2,421925$ & 2,472169 \\
\hline Temas pedagógicos en las reuniones de consejo & 2,307706 & 7,525256 & 0,7932452 & 7,249977 \\
\hline Frecuencia de visitas del director al aula & $-0,8386752$ & 1,744682 & 1,76539 & 1,824406 \\
\hline Frecuencia de visitas del supervisor a la escuela & $-1,831225$ & 1,816663 & 0,7343776 & 1,760419 \\
\hline Número de cursos de actualización & $3,400278^{* * *}$ & 0,6997372 & 0,3969596 & 0,8066556 \\
\hline Años de experiencia del profesor & 0,1394473 & 0,220062 & $1,853548^{* * *}$ & 0,2534648 \\
\hline ESCYC medio & $-2,871836$ & 3,648901 & $-6,67462$ & 3,53687 \\
\hline Involucramiento medio & $36,17314^{* * *}$ & 6,20022 & $36,39565^{* * *}$ & 7,67549 \\
\hline Beneficios de la evaluación & $-13,55397$ & 18,65418 & $-4,653373$ & 17,33982 \\
\hline Evaluación pedagógica & 2,596178 & 4,083326 & $-2,957794$ & 4,140163 \\
\hline Asistencia & $-8,445618$ & 6,054073 & $-5,204659$ & 6,414926 \\
\hline Puntualidad & 9,053086 & 6,093901 & 8,658541 & 6,477942 \\
\hline Resultados & 1,513882 & 3,313135 & 5,026421 & 3,341685 \\
\hline Constante & $451,0179 * * *$ & 21,68587 & $379,5643^{* * *}$ & 23,96353 \\
\hline Correlación &,- 4849615 & 0,0937402 & $-0,3912152$ & 0,0966228 \\
\hline $\begin{array}{l}\text { Prueba de verosimilitud de modelo multinivel vs regresión } \\
\text { lineal } \mathrm{Ch}^{2}\end{array}$ & $6.731,47^{* * *}$ & & $5.533,37 * * *$ & \\
\hline
\end{tabular}

Fuente: Elaboración propia a partir de prueba ENLACE y cuestionario CONTEXTO 2012. 


\section{Modelo de ecuaciones estructurales (SEM)}

Con la intención de contrastar la Hipótesis III en que se plantea que las variables de gestión institucional afectarían positivamente los resultados escolares por medio de impactar positivamente en el involucramiento del docente, se construyó un modelo de ecuaciones estructurales (SEM).

Como ya se señaló, en el modelo de ecuaciones estructurales se propone una relación entre la variable dependiente (promedio general en español y matemáticas en la prueba ENLACE), con el resto de las variables independientes que se analizaron en el modelo multinivel. Este modelo se resume en la Figura 1. 


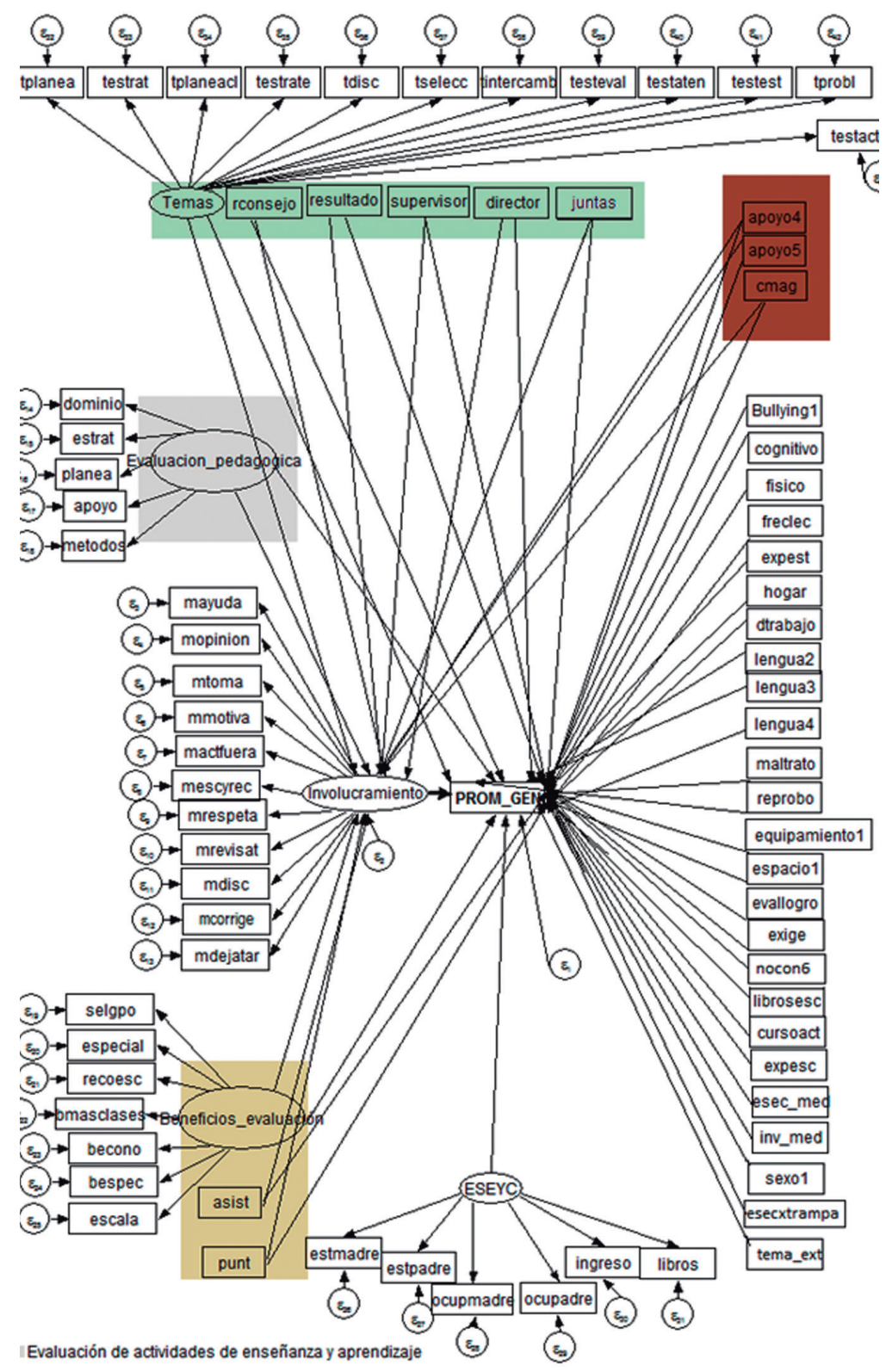

IPromueven organización y trabajo conjunto de docentes

Figura 1. Modelo de ecuaciones estructurales

Fuente: Elaboración propia a partir de prueba ENLACE y cuestionario CONTEXTO 2012. 
La regresión se puede ver expresada al observar las flechas que parten de las variables independientes y que se dirigen al cuadro donde está escrito PROM_GEN (promedio general), que es la variable dependiente.

Los modelos SEM también se componen por un CFA. Este se observa a través de las variables en un óvalo que corresponden a las variables latentes que ya se definieron anteriormente (recuérdese que no todas las variables de gestión institucional o relacionadas con esta se transformaron en índices). Las variables que son señaladas por una flecha que parten de la variable latente se refieren a los componentes de esta. Los óvalos con la letra griega $\varepsilon$ (eta) corresponden al error tanto de los modelos de regresión como los de medición (es decir, de los CFA).

El efecto indirecto se puede observar con las flechas que también se dirigen a la variable de involucramiento del docente.

En esta figura se presenta una modelación en que todas las variables independientes tienen efecto en los resultados de la prueba ENLACE en español y matemáticas. Aunque también se plantea que las variables de gestión institucional, Carrera Magisterial e involucramiento de padres de familia, afectan el logro escolar a través del involucramiento docente. Con este procedimiento se propone constatar el grado en que las variables mencionadas influyen en el promedio del niño por medio de impactar en el involucramiento del docente con sus estudiantes.

A continuación se presentan las tablas con los impactos indirectos que tienen las variables señaladas en el logro escolar (por medio del involucramiento del docente), y con las medidas de bondad de ajuste del modelo ${ }^{22}$.

22 El modelo arroja los dos tipos de efectos, directos e indirectos. El cálculo del modelo SEM se realizó sin utilizar un análisis multinivel, aunque para salvar esta dificultad se agruparon los errores por escuela. A pesar de que los resultados (efectos directos) de ambos modelos son bastante similares, debido a que únicamente el modelo de regresión de la sección anterior considera los efectos multinivel, se prefirió realizar la interpretación de los efectos directos con tal modelo. 
82 GESTIÓN INSTITUCIONAL, INVOLUCRAMIENTO DOCENTE Y DE PADRES DE FAMILIA EN ESCUELAS PÚBLICAS DE MÉXICO - C. Acevedo, G. Valenti y E. Aguiñaga

Tabla 7

Resultado del modelo de ecuaciones estructurales. Efectos indirectos sobre promedio general. Cuarto y sexto grados ${ }^{23}$

\begin{tabular}{lllll}
\hline & \multicolumn{2}{c}{ Cuarto grado } & \multicolumn{2}{c}{ Sexto grado } \\
\hline JUNTAS & Coeficiente & P-valor & Coeficiente & P-valor \\
APOY04 & 0,027 & 0,953 & 0,239 & 0,781 \\
APOY05 & $4,045^{* * *}$ & 0 & $4,984^{* * *}$ & 0 \\
CMAG & $2,285^{* * *}$ & 0 & $3,547^{* * *}$ & 0 \\
TEMA_INT & 0,033 & 0,779 & 0,212 & 0,109 \\
RCONSEJO & $-0,287$ & 0,638 & 0,966 & 0,209 \\
RESULTADO & $-0,253$ & 0,265 & $-0,261$ & 0,405 \\
SUPERVISOR & 0,322 & 0,358 & $-0,216$ & 0,575 \\
DIRECTOR & 0,018 & 0,917 & $-0,076$ & 0,699 \\
EV_PED3 & 0,248 & 0,241 & 0,153 & 0,505 \\
BENEF & $-0,165$ & 0,722 & $-0,08$ & 0,871 \\
\hline
\end{tabular}

Fuente: Elaboración propia a partir de prueba ENLACE y cuestionario CONTEXTO 2012.

Tabla 8

Medidas de ajuste del modelo de ecuaciones estructurales. Cuarto y sexto grados ${ }^{24}$

\begin{tabular}{lcc}
\hline Índices & Cuarto grado & Sexto grado \\
RMSEA & 0,039 & 0,036 \\
CFI & 0,848 & 0,873 \\
TLI & 0,862 & 0,864 \\
\hline
\end{tabular}

Fuente: Elaboración propia a partir de prueba ENLACE y cuestionario CONTEXTO 2012.

Al observar los resultados de los efectos indirectos del modelo SEM, se constata que únicamente los beneficios de la evaluación docente (BENEF) y el involucramiento de los padres en el proceso

23 Estos datos vienen dados por el cálculo realizado en Mplus, software especializado en modelos SEM. Al correr el modelo en Stata (errores agrupados por escuelas), obtuvimos variaciones menores en los coeficientes y se mantuvo la misma tendencia. A continuación presentamos los valores calculados en Stata en caso de que algún lector le interese replicar el modelo en este software. Para cuarto grado: 3,260812*** (apoyo4), 2,460487*** (apoyo5), 2,733497* (benef). Para sexto grado: 2,829694*** (apoyo4), 1,58865*** (apoyo5), 2,619051** (benef). Al igual que con Mplus, estos son los únicos coeficientes significativos.

24 Para el RMSEA valores menores a 0,05 son deseables pero menores a 0,08 son aceptables. Para el CFI y TLI se buscan valores cercanos o mayores a 0,9 . 
de enseñanza-aprendizaje que se da en la escuela (si están pendientes de las calificaciones de sus hijos o pupilos APOYO4 y asistencia a juntas escolares APOYO5) resultan significativas. Únicamente estas variables influyen de manera indirecta en el resultado del estudiante en la prueba a través de elevar el involucramiento del docente.

Por lo tanto, junto con el involucramiento de los padres de familia, solo aquellas variables de gestión institucional que se encuentran en la dimensión organizativa, y que se remiten a los beneficios individuales que recibe el docente a partir de la evaluación que realiza el director de este, tienen un efecto positivo en aumentar el involucramiento del docente y mediante este efecto, impactar positivamente en el logro escolar del estudiante.

Confirmando la experiencia en campo en las escuelas mexicanas así como los resultados de los estudios de caso realizados en Chile (Unicef y Mineduc, 2004), la participación de padres en torno al proceso de enseñanza-aprendizaje de sus hijos afecta positivamente el involucramiento del docente y mediante este, incrementa el logro escolar. Como se vio en el análisis multinivel, las variables de involucramiento de los padres en el proceso de enseñanza tenían un efecto directo positivo en los resultados escolares. Frente a los resultados del modelo SEM, ahora se puede decir que además de este efecto directo, el involucramiento de los padres o tutores en los temas escolares así como en parte del proceso que ocurre en la enseñanzaaprendizaje escolar, incentiva que los docentes se involucren en mayor medida en prácticas pedagógicas exitosas.

Ahora bien, llama la atención que la evaluación pedagógica acerca de las prácticas de enseñanza-aprendizaje que lleva a cabo el director (EV_PED3), así como aquellas actividades que podrían promover perspectivas consensuadas entre los docentes (y padres de familia) respecto del proceso de enseñanza aprendizaje (JUNTAS, TEMA_INT, RCONSEJO, RESULTADO, SUPERVISOR, DIRECTOR), especialmente, aquellas reuniones en donde se conversa en torno a temas pedagógicos (TEMA_INT), no resulten significativas para elevar el resultado escolar por medio de estimular el involucramiento del docente. De acuerdo con la literatura especializada estas variables 
deberían ser centrales en promover actitudes del profesorado que forman parte de lo que se ha denominado involucramiento docente.

Finalmente, se constata que el nivel en Carrera Magisterial (CMAG) no presenta un efecto significado directo o indirecto. Tal irrelevancia puede dar elementos para sostener los cambios que se están introduciendo en el sistema de incentivos nacional a docentes en la actual reforma educativa.

\section{Discusión}

Se confirma la Hipótesis I, a saber, que existe un efecto positivo y directo entre involucramiento de docentes y padres de familia respecto de los resultados de los estudiantes. Además, se puede concluir que el involucramiento docente se halla entre los efectos directos más importantes del conjunto de las variables revisadas.

Respecto de las variables de gestión institucional, confirmando la Hipótesis II, se constata que ninguna de estas resulta significativa en sus efectos directos.

Respecto del efecto indirecto que se planteó en la Hipótesis III, resultan significativas únicamente aquellas variables de gestión institucional que se relacionan con beneficios individuales otorgados a los docentes tales como capacidad de seleccionar a su grupo, permisos especiales, reconocimiento en la escuela, lograr más horas de clase, beneficios económicos y en especies, y obtener ascensos.

Ahora, si se considera el efecto positivo (directo e indirecto) en los resultados escolares que presenta el involucramiento de los padres (o tutores) en los temas escolares así como en parte del proceso que ocurre en la enseñanza-aprendizaje escolar; y si consideramos el elevado efecto directo del involucramiento docente (muy cercano al efecto del ESCYC): se puede decir que las desventajas sociales de los estudiantes pueden aminorarse si los padres se involucran con los docentes. Tal relación se vería potenciada en un contexto de gestión institucional directiva que apoye y reconozca la labor docente en el sentido que otorgue beneficios individuales. 
No obstante, los resultados respecto de la influencia irrelevante que tiene en las escuelas mexicanas la gestión institucional directiva de carácter cooperativa alertan sobre la necesidad de profundizar en este aspecto, sobre todo por el efecto positivo que tal gestión presenta en diversas investigaciones realizadas en una diversidad de casos y países, donde se expone la asociación positiva entre la construcción de visiones colectivas y logro académico.

Respecto de tales variables es necesario subrayar que de acuerdo con la literatura especializada, estas serían eficaces si y solo si promueven entre los agentes escolares un trabajo y visiones pedagógicas colectivas que impacten en el núcleo pedagógico (Acevedo y Valenti, 2015; Elmore, 2010; Fullan, 2010; Malone, 2011). Es decir, teniendo como objetivo impactar en el proceso de enseñanza-aprendizaje que ocurre al interior del aula, en la literatura especializada se recalcan prácticas de gestión institucional que estimulan un trabajo colectivo de construcción de visiones compartidas acerca de cómo los estudiantes aprenden de sus debilidades y fortalezas, y que se tornan en la base de acuerdos sobre cómo aminorar las primeras y potenciar las segundas. En palabras de Elmore (2010), estas prácticas se tornan efectivas cuando se dirigen en la dirección de consolidar acuerdos colectivos, superando definiciones individualistas de cómo impactar positivamente en el proceso de enseñanza-aprendizaje: un "acuerdo entre los profesores y directores acerca de los objetivos deseables y la forma de alcanzarlos", y así superar "definiciones del éxito docente y la forma de alcanzarlo" de corte "individualistas" (p. 120). En este mismo sentido es que ya a mediados de los años ochenta Rosenholtz afirmaba que "sin definiciones comunes la colaboración entre colegas y directores carece de utilidad" (Elmore, 2010, p. 120).

Confirmando este planteamiento, en los estudios de las escuelas eficaces se ha constatado que existe una serie de valores e intereses compartidos, es decir, una cultura común y consensuada respecto de las formas de diseñar e implementar prácticas pedagógicas que tengan como objetivo impactar en el núcleo pedagógico (Acevedo y Valenti, 2015). 
Tal como concluye Hattie (2009) en su estudio de más de 800 metaanálisis, se entiende que un componente clave del rol de liderazgo institucional del director consiste en crear un ambiente propicio para el trabajo colaborativo, un ambiente en donde no se tenga temor a discutir aspectos pedagógicos y a quedar expuestos a equivocarse y al error, es más: un ambiente de trabajo colectivo en donde el error sea bienvenido y visto como una oportunidad de aprendizaje. Se trata de la existencia de un liderazgo institucional efectivo que genera una situación en que los docentes se sienten seguros de llevar a cabo un proceso en que se evalúan y cuestionan sus métodos de enseñanza y en que también se comparten experiencias exitosas, todo ello a partir de evidencia basada en lo que ocurre en la clase. Aunque en la literatura no se analiza de forma explícita, se puede inferir que es necesario que se generen estas condiciones para que se dé lugar al efecto indirecto que presentan las variables de gestión institucional -de carácter cooperativo- que se plantearon en la Hipótesis III.

Sobre esta base y sumado a los datos de la investigación presentados en este estudio, adquiere sentido la hipótesis de que el impacto no significativo de las variables de gestión institucional que remiten a una acción colaborativa, estaría dando cuenta de la carencia de una gestión institucional efectiva que logre una coherencia entre objetivos y valores básicos en la comunidad de profesores. Esto, en un ambiente escolar que se caracteriza por un desequilibrio que inclina la balanza al reconocimiento y recompensa del trabajo individualista en contraposición con el trabajo colaborativo. En línea con esta hipótesis, y de nuevo para mediados de los ochenta, Rosenholtz destacaba que: "la relación entre el director y los profesores no tenía un efecto directo sobre el desempeño escolar, pero sí un efecto indirecto cuando estaba mediatizado por objetivos comunes de la escuela y por la contratación de profesores, su socialización y evaluación" (Rosenholtz, 1985, citado por Elmore, 2010, p. 120). Más recientemente, y en relación con las experiencia de las escuelas ELTO que se lleva a cabo en 
Estados Unidos ${ }^{25}$, Malone y Noam (2011) han presentado evidencia en la misma línea. Así, en los casos exitosos de ELTO, subrayan la importancia de la gestión institucional del director respecto de: generar acuerdos colectivos y consensuados sobre temas pedagógicos, contratar a profesores que comparten una visión e involucrarse directamente en el proceso de enseñanza docente ${ }^{26}$.

Sobre esta base, es posible conjeturar que se estaría dando la situación de que efectivamente en las escuelas públicas de primaria en México se llevan a cabo actividades de gestión institucional como evaluación pedagógica del trabajo del profesor y reuniones en las que se conversan temas pedagógicos, empero, no se estarían generando las condiciones de gestión institucional para impulsar compromisos en torno a visiones comunes y consensuadas que busquen impactar en el núcleo pedagógico. Así, tales actividades no se traducen en una mayor coherencia entre las prácticas de enseñanza-aprendizaje y en una mayor reflexión colectiva en torno a estas (Elmore, 2010, p. 51). Tal situación otorgaría elementos para entender que estas actividades no redunden en un mayor involucramiento docente, y a través de este, en mejores resultados académicos de los estudiantes ${ }^{27}$. En consecuencia, se estaría perdiendo el enorme potencial que para el logro escolar presentan estas variables.

25 Con ELTO se refiere a escuelas en desventaja ubicadas en Estados Unidos y que se encuentran en la modalidad de Expanded learning time and opportunities (ELTO). Esta modalidad surge a partir de la combinación entre un sistema de Expanded learning time school (ELT) y organizaciones, agencias e intermediarios basados en la comunidad que ofrecen expanded learning opportunities (ELO) —-servicios académicos y sociales en la escuela, después de la escuela, en el verano, todo el año-. Estas escuelas (inmersas en la modalidad ELTO) trabajan sobre la base de un visión colectiva que se fundamenta en: compartir datos, participar en "evaluaciones que informan sobre la práctica", y dar "cuenta de una serie de indicadores que guían el aprendizaje y éxito estudiantil" (Malone y Noam, 2011, p. 123).

26 La importancia que tiene la promoción del trabajo colectivo en la gestión institucional también se ha destacado en el ámbito universitario. En este sentido, es que Frank Rhodes, presidente de la Universidad de Cornell entre 1977-1995, señala que "el rector eficiente plasmará un nivel de energía e iniciativa, de optimismo y franqueza contagiosos. Es este espíritu, y el trabajo en equipo que promueve, lo que logra el éxito" (Rhodes, 2009, p. 294).

27 Lograr el impacto positivo en la mejora escolar de diversas variables de gestión institucional se enfatiza aún más si se tiene en cuenta que en sistemas educativos altamente segregados, los docentes que provienen de familias con estatus socioeconómico bajo, obtienen su formación inicial en instituciones de bajo prestigio académico y desarrollan su carrera laboral principalmente en escuelas que atienden mayormente a sectores desfavorecidos y que poseen carencias de todo tipo (Puga, Polanco y Corvalán, 2015). Es decir, las variables de gestión institucional se deben desarrollar en aras de elevar el desempeño docente que se lleva a cabo en condiciones altamente adversas. 
Asimismo, como un componente de la carencia de una gestión institucional efectiva, se puede poner en duda la calidad y pertinencia de la manera en que se están llevando a cabo los Consejos Técnicos Escolares y la forma en que se plantean y discuten los temas pedagógicos. Sin embargo, tales elementos así como la existencia o no de una cultura educativa consensuada y dirigida a elevar los logros en los aprendizajes del estudiante, no pueden ser estudiados en profundidad a partir de un análisis cuantitativo de bases de datos. Se hace necesario realizar una investigación cualitativa en un conjunto de escuelas analíticamente seleccionadas.

\section{Conclusiones y agenda de investigación}

Si bien en la literatura especializada se ha realizado bastante investigación sobre casos de escuelas eficaces, estos estudios generalmente adolecen de dos falencias. Por un lado, parte importante de estos "se detiene en los insumos más que en los procesos al interior de la escuela" (Unicef y Mineduc, p. 31). Por otro, estos estudios se enfocan en analizar casos únicamente exitosos y no utilizan casos de control que le otorguen validez a sus hallazgos.

Dando cuenta de estos vacíos y para identificar cómo y cuáles factores de gestión institucional aportan positivamente en la mejora educativa de estudiantes desfavorecidos (mayoría que asiste a la educación pública), es necesario conocer cómo tales factores impactan en el proceso de enseñanza que se desarrolla al interior del aula (núcleo pedagógico); y profundizar en el proceso por el cual estos factores se han construido y consolidado en las escuelas efectivas. Asimismo, es necesario contrastar estas escuelas exitosas con aquellas que sirvan como grupo de control, de modo de conseguir validez en las interpretaciones y hallazgos del estudio.

Además de los factores de gestión institucional considerados en el presente análisis, es preciso profundizar en la existencia de factores de gestión institucional eficaces muy difíciles de constatar a partir de bases de datos cuantitativas y que se han establecido como centrales en investigaciones empíricas realizadas en América Latina, Estados Unidos y Canadá (entre otros) por Coburn y Rusell (2008), Elmore 
(2010), Fullan (2010), Hattie (2009), Lee y Smith (1996), Malone (2011), Unicef y Mineduc (2004) y Treviño y Treviño (2004). Entre estos factores se encuentran:

- Gestión institucional centrada en lo pedagógico, en el aprendizaje de los estudiantes.

- Reuniones formales e informales de agentes educativos en las que se logran acuerdos pedagógicos.

- Elevada frecuencia de las relaciones entre agentes educativos, confianza en estas relaciones, acceso a la información, y profundidad y congruencia de la interacción (es decir, se debaten temas pedagógicos y se logran consensos al respecto).

- Existencia de proyecto educativo compartido.

- Consolidación de una visión común sobre los aprendizajes.

- Conocimiento de parte de los directivos de las fortalezas y debilidades de los profesores.

- Apertura para la flexibilidad y creatividad del trabajo de los profesores.

- Capacitación constante de los profesores y socialización del conocimiento.

- Elevada preocupación por la planificación y evaluación del trabajo docente.

- Inserción de las escuelas en redes que les permiten cubrir necesidades (otros colegios, universidades, centros de salud, ONG, etc.).

- Integración de las escuelas con sus entornos locales (colaboración para solucionar problemas de la comunidad, creaciones de actividades artísticas, actividades de formación, etc.).

- Acciones de integración de padres o tutores a actividades escolares y en la formación de sus hijos o pupilos.

- Liderazgo distribuido.

- Liderazgo efectivo del director o culture change principal ${ }^{28}$.

28 Las cualidades de liderazgo efectivo han adquirido forma en la figura que Fullan denomina Culture Change Principal (Fullan, 2002), a saber: un líder directivo con elevado grado de manejo del conocimiento, de las emociones, de la construcción de coherencia entre las acciones de los distintos agentes escolares y que promueve el fortalecimiento de sus relaciones (Fullan, 2002; Unicef y Mineduc, 2004). 
Al estudiar los procesos de construcción de los factores de éxito escolar es importante considerar que en tanto sistema político, el sistema educativo y las escuelas que forman parte de este están cruzados por relaciones de conflicto y cooperación (Bonal, 1998; Street, 1984). Es en este marco en donde se llevan a cabo negociaciones. Descuidar estos elementos políticos ha debilitado la capacidad comprensiva de la sociología de la educación (Bonal, 1998).

Asimismo, la atención en las negociaciones que se llevan a cabo en el ámbito escolar dirige la mirada a los valores e intereses que defienden los agentes educativos, pues "las creencias y valores de los docentes" son los que los que los movilizan para lograr diversos tipos de acuerdos (Coburn \& Rusell, 2008, p. 207).

No se debe perder de vista, entonces, que el sentido de los agentes así como el proceso por el cual se ha construido y consolidado la gestión institucional eficaz, resultan pertinentes en tanto el quehacer docente y directivo se enmarca en una apropiación particular de una forma de gestión institucional que pone en juego valores, intereses y negociaciones, que configuran disputas irreconciliables o la construcción de acuerdos (Anderson, 2003; Coburn \& Rusell, 2008).

Desde esta perspectiva, y teniendo como base el estudio presentado, se constata la necesidad de analizar en las escuelas "la construcción de equilibrios y negociaciones entre grupos de interés contrapuestos que expliquen tanto el proceso de generación de políticas como su proceso de aplicación" (Bonal, 1998, p. 191). Esto, en un contexto en que recientemente el estudio de los procesos escolares ha sido una preocupación de política educativa subrayada por el INEE: "Los datos indican la necesidad de desarrollar mecanismos educativos que promuevan ambientes de convivencia, de climas escolares y de aula positivos tanto para propiciar condiciones favorables a la enseñanza y al aprendizaje, como para asegurar el respeto a los derechos humanos de todos los miembros de la comunidad escolar" (2014, p. 76). 


\section{Referencias}

Acevedo, C. y Valenti, G. (2015). Propuesta analítica para estudiar innovaciones culturales y políticas. En G. Valenti (Coord.), Hacia una nueva cultura educativa de los sistemas educativos estatales (pp. 19-65). México D.F.: FLACSO-México.

Anderson, S. (2003). The school district role en educational change: A review of the literature (ICEC Working Paper, 2). Ontario: Ontario Institute for Studies in Education.Recuperado de: http://fcis.oise.utoronto.ca/ icec/ workpaper2.pdf

Bernstein, B. (1971). Class, Codes and Control. Volume 1: Theoretical Studies Towards A Sociology of Language. London and New York: Routledge.

Blanco, E. (2009). Eficacia escolar y clima organizacional: apuntes para una investigación de procesos escolares. Estudios Sociológicos, 80(27), 671694.

Bonal, X. (1998). Sociología de la educación. Barcelona: Paidós.

Bourdieu, P. (1998). The state nobility: Elite schools in the field of power. Stanford: University Press.

Bourdieu, P. \& Passeron, J. (1970). La reproduction. París: Les Éditions de Minuit.

Bracho, T. (2009). Innovación en la política educativa. México D.F.: FLACSOMéxico.

Branch, G., Hanushek, E., \& Rivkin, S. (2013). School leaders matter: Measuring the impact of effective principals. Education Next, 1(13), 62-69.

Brunner, J. J. y Elacqua, G. (2003). Factores que inciden en una educación efectiva. Recuperado de: http://ww2.educarchile.cl/UserFiles/P0001/ File/Factores\%20q\%20inciden $\% 20$ en $\% 20$ una $\% 20$ educacion $\% 20$ efectiva_2004.pdf

Coburn, C. \& Rusell, J. (2008). District policy and teachers's social networks. Educational Evaluation and Policy Analysis, 3(30), 203-235. https://doi.org/10.3102/0162373708321829

Coleman, J. (1966). Equality of educational opportunity. Washington: University of South Carolina, Government Information and Maps Departament.

Elmore, R. (2010). Mejorando la escuela desde la sala de clases. Santiago de Chile: Fundación Chile. 
Fernández, T. (2004). De las escuelas eficaces a las reformas educativas de segunda generación. Estudios Sociológicos, 2(23), 377-408. Recuperado de: http://www.redalyc.org/articulo.oa?id=59806505

Fierro, C., Tapia, G., y Rojo, F. (2009). Descentralización educativa en México. Un recuento analítico. París: OCDE.

Fondo de Naciones Unidas para la Infancia, Unicef y Ministerio de Educación, Mineduc, Chile. (2004). Escuelas efectivas en sectores de pobreza. Santiago de Chile: Fondo de Naciones Unidas para la Infancia, Unicef.

Fuentes, O. (2013). Las tareas del maestro y los desafíos de la evaluación docente. En R. Ramírez (Coord.), La reforma constitucional en materia educativa: alcances y desafíos (pp. 17-34). México D.F.: Senado de la República-Instituto Belisario Domínguez.

Fullan, M. (2002). Principals as leaders in a culture of change. Recuperado de: http://michaelfullan.ca/wp-content/uploads/2016/06/13396053050. pdf

Fullan, M. (2010). The role of the district in tri level reform. En P. Peterson, E. Baker, \& B. McGaw (Eds.), International Encyclopedia of Education, vol. 6, (pp. 295-302). Oxford: Elservier.

Fuller, B. \& Clarke, P. (1994). Raising school effects while ignoring the culture? Local conditions and the influence of classroom tools, rules and pedagogy. Review of Educational Research, 1(64), 119-157. https://doi.org/10.2307/1170747

Hanushek, E. (1992). The trade-off between child quantity and quality. Journal of Political Economy, 1(100), 84-117. https://doi.org/10.1086/261808

Hattie, J. (2009). Visible learning. A synthesis of over 800 meta-analyses relating to achievement. London and New York: Routledge.

Heath, S.B. (1983). Ways with words: Language, life, and work in communities and classrooms. Cambridge: Cambridge University Press.

Instituto Nacional para la Evaluación Educativa, INEE. (2014). El derecho a una educación de calidad, Informe 2014. México D.F.: Autor.

Laboratorio Latinoamericano de Evaluación de la Calidad de la Educación, LLECE. (2000). Primer estudio internacional comparativo sobre lenguage, matemática y factores asociados. Segundo informe, (First International Comparative Study about Language, Mathematics and Associated Factors. Second Report). Santiago de Chile: Unesco-Santiago.

Lee V. \& Smith, J. (1996). Collective responsibility for learning and its effects on gains in achievement for early. American Journal of Education, 2(104), 103-147. https://doi.org/10.1086/444122 
López, N. (2006). Educación y desigualdad social. Buenos Aires: Ministerio de Educación, Ciencia y Tecnología de la República Argentina.

Malone, H. (2011). Building an expanded learning time and opportunities school: Principals' perspectives. New Directions for Youth Development, 131, 107-117. Recuperado de: https://doi.org/10.1002/yd.412

Malone, H. \& Noam, G. (2011). Next Steps en the Expanded Learning Discourse. New Directions for Youth Development, 131, 119-135. http:// onlinelibrary.wiley.com/doi/10.1002/yd.413/abstract

Martinic, S. (2010). La evaluación y las reformas educativas en América Latina. RIEE, 3(3), 31-43.

McCulla, N. \& Degenhardt, L. (2015). Journeys to school leadership. How action learning identified what participants valued in a year-long Australian leadership development program centered on principles of good practice. Educational Management Administration \& Leadership, 4(44), 558-577. https://doi.org/10.1177/1741143214558574

Méndez, I. (2003). Modelos estructurales de covarianza. En G. L. Daniel (Ed.), Modelamiento estructural en las ciencias sociales, (pp. 13-36). Hermosillo, Sonora: Universidad de Sonora.

Nye, B., Konstantopoulos, S., \& Hedges, L. (2004). How large are teacher effects? Educational Evaluation and Policy Analysis, 3(26), 237-25. https://doi.org/10.3102/01623737026003237

PISA (2000). Knowledge and skills for life. First results from PISA 2000. Recuperado de: https://www.oecd.org/edu/school/programmeforint ernationalstudentassessmentpisa/33691620.pdf

Organisation for Economic Co-operation and Development, OECD (2012), Equity and quality in education: Supporting disadvantaged students and schools. Recuperado de: http://www.oecd.org/edu/school/equityand qualityineducationsupportingdisadvantagedstudentsandschools.htm

Ortíz, I. (2015). Escuelas inclusivas en el contexto de segregación social del sistema escolar chileno. Calidad en la Educación, 42, 93-122. https:// doi.org/10.4067/s0718-45652015000100004

Puga, I., Polanco, D., y Corvalán, D. (2015). Segregación de la formación y carrera docente y su rol en la reproducción social de la desigualdad. Calidad en la Educación, 42, 57-102. https://doi.org/10.4067/s0718-45652015000200003

Raykov, T. \& Marcoulides, G. A. (2006). A first course in structural equation modeling, Mahwah, NJ: Lawrence Erlbaum. 
Reynolds, A. \& Walberg, H. (1992). A structural model of science achievement and attitude: An extension to high school. Journal of Educational Psychology, 3(84), 371-382. https://doi.org/10.1037//0022-0663.84.3.371

Rhodes, F. (2009). La creación del futuro: La función de la universidad norteamericana. Buenos Aires: Fundación Universidad Palermo.

Rivkin, S. (2005). Teachers, schools, and academic achievement. Econometric Society, 2(73), 417-458.

Rubio, M. (2011). Diplomado en gestión institucional e innovación educativa (DGIIE). Guía de estudio. México D.F.: FLACSO, México.

Salazar, R., Flores, U., Florez, N., Luna, M., y Valenti, G. (2010). Desempeño escolar México 2010. Un enfoque en la calidad con equidad (Reporte de investigación). México D.F.: FLACSO-México.

Sanders, W. \& Rivers, J. (1996). Cumulative and residual effects of teachers on future student academic achievement. (Research Progress Report). Knoxville, TN: University of Tennessee Value-Added Research and Assessment Center.

Schacter, J. \& Thum, Y. (2003). Paying for high- and low-quality teaching. Economics of Education Review, 23, 411-430. https://doi.org/10.1016/j.econedurev.2003.08.002

Scheerens, J. (2000). Improving school effectiveness. Paris: Fundamental of education planning-IIEP.

Scheerens, J. \& Bosker, R. (1997). The foundations of educational effectiveness. International, Review of Education - Internationale Zeitschrift für Erziehungswissenschaft - Revue Internationale de l'Education, 45(1), 113-119.

Schmelkes, S. (1997). Evaluación del aprendizaje y calidad de la educación básica. En Problemas y políticas de la Educación básica. Programa y materiales de apoyo para el estudio. 1er Semestre. Licenciatura en Educación Primaria (pp. 95-106). México D.F.: SEP.

Street, S. (1984). Los distintos proyectos para la transformación del aparato burocrático de la SEP. Perfiles Educativos, 7, 14-29.

Tapia, A. y Valenti, G. (2015). Desigualdad educativa y desigualdad social en México. Nuevas evidencias desde las primarias generales de los estados. Revista Perfiles Educativos IIESU-UNAM, 151(38), 32-54.

Treviño, E. y Treviño, G. (2004). Estudio sobre las desigualdades educativas en México: la incidencia de la escuela en el desempeño académico de 
los alumnos y el rol de los docentes (Informe de investigación para el Instituto Nacional para la Evaluación Educativa). México, D.F.: INEE.

Watkins, T. (1997). Teacher communications, child achievement, and parent traits in parent involvement models. Journal of Educational Research, 1(91), 3-14. https://doi.org/10.1080/00220679709597515

Winters, M. (2012). Teachers matter. Lanham, MD: Rowman \& Littlefield Publishers.

Recibido: 22 de junio de 2016

Aceptado: 26 de enero de 2017 